\title{
Validation of the Replica Trick for Simple Models
}

\author{
Takashi Shinzato* \\ Mori Arinori Center for Higher Education and Global Mobility, Hitotsubashi University, Tokyo, 1868601, Japan.
}

(Dated: December 27, 2017)

\begin{abstract}
We discuss replica analytic continuation using several simple models in order to prove mathematically the validity of replica analysis, which is used in a wide range of fields related to large scale complex systems. While replica analysis consists of two analytical techniques, the replica trick (or replica analytic continuation) and the thermodynamical limit (and/or order parameter expansion), we focus our study on replica analytic continuation, which is the mathematical basis of the replica trick. We apply replica analysis to solve a variety of analytical models, and examine the properties of replica analytic continuation. Based on the positive results for these models we propose that replica analytic continuation is a robust procedure in replica analysis.
\end{abstract}

PACS number(s): 89.90.+n, 75.50.Lk

\section{INTRODUCTION}

Replica analysis is a widely used analytic approach, developed for the analysis of large scale complex systems in cross-disciplinary fields. Applications of replica analysis include the analysis of the physical properties of disordered magnetic materials, evaluation of the learning performance of neural networks, estimation of the transmission rate of channel coding and optimization of the investment risk of diversification investments [1-13]. Replica analysis has a long history and has been discovered (and rediscovered) in several research fields [14-20]. In one of the pioneering works on replica analysis, Edwards et al. investigated the singular properties of disordered magnetic materials [4], generally called spin glass. They first derived and used replica analysis to examine the physical properties of spin glass. Based on the observation that 2-body interactions between localized spins can be modeled by the Ruderma-Kittel-Kasuya-Yoshida interaction, they assumed that 2-body interactions between localized spins can be randomly assigned and can be used to analyze the singular physical properties of spin glass. Subsequently, Sherrington et al. developed this spin glass model into a fully connected spin glass model by applying the mean field approximation, since it is well known that solutions based on the mean field approximation are the most rigorous $[5,6]$.

Recently, following its success in the analysis of the physical properties of spin glass, replica analysis has been applied to the mathematical structures and mathematical analogies of other large scale complex systems. For instance, for the analysis of the learning performance of neural networks, Amit et al. applied replica analysis to the associative storage model and examined the mechanism for recalling stored memories, and evaluated the learning capacity of the associative storage model $[7,8]$. Further, Gardner investigated the learning performance of perceptron, one of the most simplified neural network

*takashi.shinzato@r.hit-u.ac.jp models, using replica analysis, and found that it is consistent with the findings based on combinatorics in the previous work of Cover et al. [9, 21, 22]. As part of an evaluation of the transmission rate of communication technology, Kabashima et al. used a sparse connected spin glass model to resolve the decoding problem of the binary linear Low Density Parity Check code (LDPC) and guarantee that the communication performance of its linear code is close to the Shannon limit using replica analysis [10]. In addition, Tanaka used the posterior probability as a type of perceptron learning to resolve the maximizer of the posterior marginal estimation problem for the third generation of radio communication technology Code Division Multiple Access (CDMA) and analyzed the bit error rate and channel capacity of CDMA using replica analysis [11]. To assess the risk of diversification investments, Ciliberti et al. dissected the minimal investment risks of the absolute deviation model and expected shortfall model in the zero temperature limit using replica analysis [12]. In a later work, Shinzato proved that the minimal investment risk and the investment concentration of the mean variance model are satisfied by the property of self-averaging using replica analysis and the Chernoff inequality [13].

Replica analysis consists of two main steps, the replica trick operation and the application of the thermodynamical limit. While several previous works have verified the effectiveness of replica analysis, in our own use of the replica trick, for convenience of analysis, we use a calculation technique where a number which is assumed to be an integer is replaced by a real or complex number in order to implement the limit operation, and for this reason replica analysis is not always guaranteed mathematically. With this in mind, we compared the results estimated by replica analysis and other approaches such as the Markov chain Monte Carlo method and steepest descent method, and found that the effectiveness of replica analysis was partly validated [23]. However, in several previous works, problems have arisen regarding negative entropy and the convexity of the order parameter expansion of free energy. To address these problems, the results of replica analysis were refined by the 1st step (or full step) replica 
symmetry breaking solution or Almeida-Thouless analysis, an approach that can provide a partial fix, but does not necessarily verify the universal reliability of replica analysis $[1,2,24-26]$. With respect to these efforts to refine replica analysis, Ogure et. al. analytically evaluated the expectation of the complex power of the partition function of the grand canonical discrete random energy model using complex analysis, and then compared three solutions obtained from replica analysis, the paramagnetic solution, ferromagnetic solution, and first replica symmetry breaking solution (spin glass solution) [27, 28]. Further, they derived the cases where the three solutions, composed of the $n$th moments of the partition function, are not analytic at $n=n_{c} \in(0,1)$ ( $n$ is called the replica number), and verified their results using numerical simulations. In addition, Tanaka rearranged the problem implied in replica analysis using the framework of the moment problem, examining several counterexamples of the moment problem and summarizing the sufficient condition that the distribution of the partition function is uniquely determined from the moment sequences of the partition function [29]. Previous works have shown that we need to analyze the Helmholtz free energy in a large scale complex system in order to implement a quenched analysis of the system. Below we discuss the mathematical basis of the expectation of the logarithm of the partition function for the analysis of Helmholtz free energy. One of the disputed issues in replica analysis is the assumption that for an integer replica number there is an analytical continuation to the real or complex number case, as this is not always valid for replica numbers near 0 . In order to validate the reliability of replica analysis mathematically, we need to examine the mathematical structure of replica analysis through the models that can be applied to the two distinct approaches for analyzing the $n$th moment of the partition function in a similar way to the argument discussed by Ogure et al.

In the present work, as the first step for resolving the validity of replica analysis and rigorously interpreting the findings obtained from replica analysis, we separate the two distinct analytical approaches, (1) the replica trick and (2) the thermodynamical limit (and/or the order parameter expansion), and focus our study on the first of the techniques, the replica trick, or equivalently replica analytic continuation. Namely, our goal in this paper is to discuss the properties of replica analysis by examining replica analytic continuation.

This paper is organized as follows. In the following section, we explain the analytical techniques of replica analysis, the replica trick and replica analytic continuation, and consider the importance of the order of integration of two distinct variables. In Section III we test replica analytic continuation using several simple models and propose a conjecture regarding replica analytic continuation. The final section is devoted to a summary and plans for future work.

\section{FROM REPLICA TRICK TO REPLICA ANALYTIC CONTINUATION}

\section{A. Why Do We Need Replica Analysis?}

Based on several studies of the disordered magnetic material model, which is represented by the EdwardsAnderson model and the Sherrington-Kirkpatrik model $[4,5]$; the neural network model, which is represented by the Hopfield model and perceptron model [7-9]; the channel coding model, which is represented by LDPC and CDMA [10,11]; and the portfolio optimization problem, which is represented by the mean-variance model and absolute deviation model $[12,13]$, it is well known that we can examine some indicators which characterize a system, for instance, the average magnetization, learning rate, and encoding rate, by evaluating the Helmholtz free energy of the system $F=-\frac{1}{\beta} \log Z$ or its expectation $E[F]=-\frac{1}{\beta} E[\log Z]$, where $\beta$ is the inverse temperature and $Z$ is the partition function. In several analyses of quenched disordered systems, replica analysis has been used constructively to assess the expectation of the Helmholtz free energy. However, the validity of several methods used in replica analysis have not been guaranteed theoretically. For this, by comparing the results obtained by replica analysis and other approaches, such as the Markov chain Monte Carlo method and the steepest descent method, we can partly verify the validity of replica analysis. In this work, as a first step for confirming the effectiveness of replica analysis, we examine the replica trick, one of the two analytical approaches which constitute replica analysis.

\section{B. The Replica Trick}

In general, the replica trick consists of the following identity equation for any positive random variable $Z$,

$$
\log Z=\lim _{n \rightarrow 0} \frac{Z^{n}-1}{n} .
$$

If the random variable $Z$ is positive and its probability density function $P(Z)$ is known, using the replica trick and the expectation of the $n$th power of $Z, E\left[Z^{n}\right]=$ $\int_{0}^{\infty} d Z P(Z) Z^{n}$, the expectation of the logarithm of $Z$, $E[\log Z]=\int_{0}^{\infty} d Z P(Z) \log Z$, is estimated as follows:

$$
E[\log Z]=\lim _{n \rightarrow 0}\left\{\begin{array}{l}
\frac{E\left[Z^{n}\right]-1}{n} \\
\frac{1}{n} \log E\left[Z^{n}\right] \\
\frac{\partial}{\partial n} \log E\left[Z^{n}\right]
\end{array},\right.
$$

where the three terms on the right hand side are consistent with each other, which can be easily confirmed by using L'Hôpital's rule. $n$ of $E\left[Z^{n}\right]$ is the replica number. However, a necessary condition for Eq. (2) is that $n$ must be a real or complex number. In other words the result obtained for $n \in \mathbf{Z}$ is regarded as $n \in \mathbf{R}$ and/or $n \in \mathbf{C}$. However, the validity of this assumption with respect to 
analytic continuation of the replica number is not always guaranteed.

It should be noted that in general if we can accurately assess $E\left[Z^{n}\right]$ without approximation when the replica number is real or complex, then we can assess $E[\log Z]$ using Eq. (2), and therefore we are not limited to analyzing quenched disordered systems. In this case, there is no question of the validity of the replica trick of Eq. (2). We consider that the assumption that the result for $E\left[Z^{n}\right]$ in $n \in \mathbf{Z}$ can be solved using the thermodynamical limit and expanding the Helmholtz free energy on order parameters and its approximation can be regarded as the result for $E\left[Z^{n}\right]$ in $n \in \mathbf{R}$ or $n \in \mathbf{C}$ is the basis of the ambiguity in replica analysis.

\section{Two Types of Integral Assessments}

In the analysis of quenched disordered systems, which are represented by the Sherrington-Kirkpatrick model in spin glass theory and the Hopfield model in associative memory, it is well-known that by analyzing the expectation of the Helmholtz free energy $F=-\frac{1}{\beta} \log Z$, $E[F]=-\frac{1}{\beta} E[\log Z]$, we can assess the typical behavior of the system. To average the free energy, we use the replica trick of Eq. (2) and analytically evaluate the $n$th power function of the partition function, $E\left[Z^{n}\right]$, in the right hand side of Eq. (2). This requires the evaluation of two distinct integrals of the internal variable of the partition function, $S$, and the external variable of the partition function, $J$. Here, we consider the relationship between the order of the two integrations of $E\left[Z^{n}\right]$ and $n$.

We first consider the procedure first-S-next-J (hereafter abbreviated FSNJ) for which when evaluating $E\left[Z^{n}\right]$, the integral or sum of the thermodynamical variable or internal variable of the partition function $S$ is first implemented, followed by the integral or sum (or the expectation) over the configuration variable or external variable of the partition function $J$. Namely, for FSNJ the expectation of $Z^{n}, E\left[Z^{n}\right]$, is derived as follows:

$$
E\left[Z^{n}\right]=\sum_{J} P(J)\left(\sum_{S} e^{-\beta \mathcal{H}(S, J)}\right)^{n},
$$

where $\mathcal{H}(S, J)$ is the Hamiltonian of the system, $Z=$ $\sum_{S} e^{-\beta \mathcal{H}(S, J)}$ is the partition function of the canonical ensemble of the inverse temperature $\beta, \sum_{J}$ and $\sum_{S}$ are the integrals or sums over the whole configuration of $J$ and $S$, respectively, and $P(J)$ describes the probability function of the external variable $J$. Since the integral or sum with respect to the internal variable $S$ is implemented first, we allow that the replica number $n$ in Eq. (3) is regarded as a real or complex number.

In the alternate procedure first-J-next-S (FJNS), the integral or sum of the external variable of the partition function is first implemented, followed by the integral or sum of the internal variable. That is,

$E\left[Z^{n}\right]=\sum_{S_{1}} \sum_{S_{2}} \cdots \sum_{S_{n}}\left(\sum_{J} P(J) e^{-\beta \sum_{a=1}^{n} \mathcal{H}\left(S_{a}, J\right)}\right)$.

We first evaluate Eq. (4) with respect to the external variable $J$ and next evaluate it with respect to the internal variables $S_{1}, \cdots, S_{n}$. The intuitive advantage of FJNS is that for any $A, B>0$, the factor expansion $(A+B)^{2.1}$ can be described by the infinite series expansion and $(A+B)^{2}<(A+B)^{2.1}<(A+B)^{3}$ holds, that is, since $(A+B)^{n}$ is described as a finite series expansion when the power number is an integer, we can evaluate each term of $(A+B)^{2}=A^{2}+2 A B+B^{2}$ and $(A+B)^{3}=A^{3}+3 A^{2} B+3 A B^{2}+B^{3}$ and then approximately assess $(A+B)^{2.1}$ using a sort of false position method from the results of $(A+B)^{2}$ and $(A+B)^{3}$. A peculiarity of this method is that if the accuracy of the approximation is reduced, negative entropy and/or convexity of the order parameter expansion of the Helmholtz free energy may occur, casting doubt on the veracity of the replica analysis.

\section{Formulation of Replica Analytic Continuation}

We set the expectation of the $n$th power of the partition function derived by FSNJ as $F(n)=E\left[Z^{n}\right],(n \in \mathbf{C})$ and for FJNS as $G(n)=E\left[Z^{n}\right],(n \in \mathbf{Z})$, shown in Fig. 1. From Eq. (4), the assessment of $G(n)$ when $n \in \mathbf{Z}$ requires changing the order of the two integrations.

If we regard $G(n)$ as an analytical function with respect to $n$ and assume that $G(n)$ can also be described as an analytical function of $n$ when $n \notin \mathbf{Z}$, then $F(n)=G(n)$ always holds in the case $n \in \mathbf{Z}$. We can then propose that this system demonstrates replica analytic continuation. By contrast, for $F(n)=G(n),(n \in \mathbf{Z})$ and $F(n) \neq G(n),(n \notin \mathbf{Z})$, the system does not satisfy replica analytic continuation. For instance, when $n \in \mathbf{C}$, $F(n)=E\left[Z^{n}\right]=A n^{2}+B n+C+D \sin (2 \pi n)$ (where $D \neq 0)$ or $n \in \mathbf{Z}, G(n)=E\left[Z^{n}\right]=A n^{2}+B n+C$, since

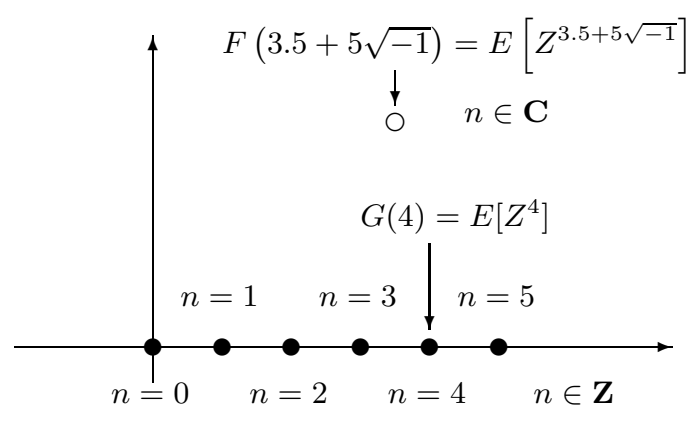

FIG. 1. Relationship between $F(n)=E\left[Z^{n}\right],(n \in \mathbf{C})$ for FSNJ and $G(n)=E\left[Z^{n}\right],(n \in \mathbf{Z})$ for FJNS on the complex plane. 
$F(n) \neq G(n),(n \notin \mathbf{Z})$, replica analytic continuation does not apply.

If replica analytic continuation holds for the system under consideration, from the replica trick of Eq. (2), the expectation of the logarithm of the partition function, $E[\log Z]$, can be accurately analyzed. We examine replica analytical continuation by applying it to physical or statistical models which can be evaluated for both the FSNJ and FJNS approaches, so that we can compare the results of the two approaches. We have selected models for which the thermodynamical limit need not be applied, so that we can test the accuracy of replica analytical continuation alone, as part of our goal to focus on the replica trick. Note that several previous works have used $G(n)=E\left[Z^{n}\right],(n \in \mathbf{Z})$, for which it is important to estimate $E[\log Z]$ accurately, and hence we need to consider the accuracy of $n$ in the neighborhood of 0 . However, we do not try to evaluate the expectation of the logarithm of the partition function consistently, since we are interested mainly in the issue of replica analytic continuation, which is not limited to the neighborhood $n=0$.

\section{SIMPLE EXAMPLES}

\section{A. Classical Harmonic Oscillator Model with Rayleigh Distribution}

First we discuss whether replica analytic continuation holds by applying the well known analytical model of the classical harmonic oscillator model. The Hamiltonian of the classical harmonic oscillator model, $\mathcal{H}(p, q)$, is defined as

$$
\mathcal{H}(p, q)=\frac{p^{2}}{2 m}+\frac{m \omega^{2}}{2} q^{2}
$$

where $p$ and $q$ are the momentum and the position of the harmonic oscillator, respectively, $m$ is the mass of the particle and $\omega$ is the angular frequency of the harmonic oscillator. The partition function $Z$ is solved as follows:

$$
\begin{aligned}
Z & =\frac{1}{h} \int_{-\infty}^{\infty} d p d q e^{-\beta \mathcal{H}(p, q)} \\
& =\frac{1}{\hbar \beta \omega}
\end{aligned}
$$

using the Dirac constant $\hbar=\frac{h}{2 \pi}$. Since the partition function of this model is analytically and easily evaluated, it is suitable for discussing the mathematical structure of replica analytic continuation.

We assume that the angular frequency of the harmonic oscillator which models the vibration phenomena $\omega$ has a Rayleigh distribution:

$$
P(\omega)=\left\{\begin{array}{ll}
\omega e^{-\frac{\omega^{2}}{2}} & \omega>0 \\
0 & \text { otherwise }
\end{array} .\right.
$$

From this, the FSNJ case with $n \in \mathbf{C}$ can be analyzed directly. The $n$th moment of $Z, F(n)=E\left[Z^{n}\right]$, is derived as

$$
\begin{aligned}
E\left[Z^{n}\right] & =\int_{0}^{\infty} d \omega P(\omega)\left(\frac{1}{\hbar \beta \omega}\right)^{n} \\
& =\frac{2^{-\frac{n}{2}}}{(\hbar \beta)^{n}} \Gamma\left(1-\frac{n}{2}\right) .
\end{aligned}
$$

Note that hereafter the Gamma function $\Gamma(s)=$ $\int_{0}^{\infty} d t t^{s-1} e^{-t},(s \in \mathbf{C})$ is often used in our discussion [30-32]. It is a meromorphic function and has a pole at the origin and at negative integer numbers, so if $F(n)=E\left[Z^{n}\right]$ is described using the Gamma function, then we will omit $n \in \mathbf{C}$ which is related to a pole of Gamma in the discussion on replica analytic continuation.

For the FJNS case, since we can permutate the integral order, the $n$th moment of $Z, G(n)=E\left[Z^{n}\right]$, is calculated as

$$
\begin{aligned}
E\left[Z^{n}\right]= & \frac{1}{h^{n}} \int_{0}^{\infty} d \omega P(\omega) \int_{-\infty}^{\infty} \prod_{a=1}^{n} d p_{a} d q_{a} \\
& \exp \left(-\frac{\beta}{2 m} \sum_{a=1}^{n} p_{a}^{2}-\frac{\beta m \omega^{2}}{2} \sum_{a=1}^{n} q_{a}^{2}\right) \\
= & \frac{1}{h^{n}}\left(\frac{2 \pi m}{\beta}\right)^{\frac{n}{2}} \int_{-\infty}^{\infty} \prod_{a=1}^{n} d q_{a} \frac{1}{1+\beta m \sum_{a=1}^{n} q_{a}^{2}} \\
= & \left(\frac{2 \pi}{h^{2} \beta^{2}}\right)^{\frac{n}{2}} \int_{-\infty}^{\infty} \prod_{a=1}^{n} d r_{a} \frac{1}{1+\sum_{a=1}^{n} r_{a}^{2}} \\
= & \left(\frac{2 \pi}{h^{2} \beta^{2}}\right)^{\frac{n}{2}} \frac{2 \pi^{\frac{n}{2}}}{\Gamma\left(\frac{n}{2}\right)} \int_{0}^{\infty} d R \frac{R^{n-1}}{1+R^{2}}
\end{aligned}
$$

In addition, we substitute the identity

$$
\begin{aligned}
2 \int_{0}^{\infty} d R \frac{R^{n-1}}{1+R^{2}} & =2 \int_{0}^{\frac{\pi}{2}} d \theta \tan ^{n-1} \theta \\
& =B\left(\frac{n}{2}, 1-\frac{n}{2}\right) \\
& =\frac{\Gamma\left(\frac{n}{2}\right) \Gamma\left(1-\frac{n}{2}\right)}{\Gamma\left(\frac{n}{2}+1-\frac{n}{2}\right)}
\end{aligned}
$$

into Eq. (9), giving

$$
E\left[Z^{n}\right]=\frac{2^{-\frac{n}{2}}}{\hbar^{n} \beta^{n}} \Gamma\left(1-\frac{n}{2}\right)
$$

where $r_{a}=q_{a} \sqrt{\beta m}, R^{2}=\sum_{a=1}^{n} r_{a}^{2}$ and $B(p, q)=$ $\frac{\Gamma(p) \Gamma(q)}{\Gamma(p+q)}$ are used. Furthermore, with respect to the integral domain $\mathcal{D}=\left\{\left(r_{1}, \cdots, r_{n}\right) \mid \sum_{a=1}^{n} r_{a}^{2}<A^{2}\right\}$, from the identical integral

$$
\int_{\mathcal{D}} \prod_{a=1}^{n} d r_{a} f\left(\sum_{a=1}^{n} r_{a}^{2}\right)=\frac{2 \pi^{\frac{n}{2}}}{\Gamma\left(\frac{n}{2}\right)} \int_{0}^{A} d R R^{n-1} f\left(R^{2}\right)
$$

the $n$-dimensional integral of $r_{1}, \cdots, r_{n}$ can be rewritten as an $n$-dimensional hyperspherical volume integral. 
From this, we find that $F(n)=E\left[Z^{n}\right],(n \in \mathbf{C})$ in Eq. (8) derived by FSNJ is consistent with $G(n)=E\left[Z^{n}\right],(n \in$ $\mathbf{Z}$ ) in Eq. (11) by FJNS. Namely, it is verified that replica analytic continuation holds for the classical harmonic oscillator.

\section{B. Classical Harmonic Oscillator Model with Positive Gaussian Distribution}

Similar to our discussion for the classical harmonic oscillator, we now discuss the case where the angular frequency $\omega$ is drawn from a positive normal distribution, $P_{\sigma}(\omega)$, defined as

$$
P_{\sigma}(\omega)=\left\{\begin{array}{ll}
\frac{2}{\sqrt{2 \pi \sigma^{2}}} e^{-\frac{\omega^{2}}{2 \sigma^{2}}} & \omega>0 \\
0 & \text { otherwise }
\end{array} .\right.
$$

For FSNJ, when $n \in \mathbf{C}$, the expectation of the $n$th power of $Z, F(n)=E\left[Z^{n}\right]$, can be assessed directly:

$$
\begin{aligned}
E\left[Z^{n}\right] & =\int_{0}^{\infty} d \omega P_{\sigma}(\omega)\left(\frac{1}{\hbar \beta \omega}\right)^{n} \\
& =\frac{2^{-\frac{n}{2}}}{(\hbar \beta \sigma)^{n} \sqrt{\pi}} \Gamma\left(\frac{1-n}{2}\right) .
\end{aligned}
$$

For FJNS, when $n \in \mathbf{Z}$, since we can permutate the integral order, $G(n)=E\left[Z^{n}\right]$ is assessed as

$$
\begin{aligned}
E\left[Z^{n}\right] & =\frac{1}{h^{n}}\left(\frac{2 \pi m}{\beta}\right)^{\frac{n}{2}} \int_{-\infty}^{\infty} \prod_{a=1}^{n} d q_{a} \\
& \int_{0}^{\infty} d \omega \frac{2}{\sqrt{2 \pi \sigma^{2}}} e^{-\frac{\omega^{2}}{2}\left(\frac{1}{\sigma^{2}}+\beta m \sum_{a=1}^{n} q_{a}^{2}\right)} \\
& =\frac{(2 \pi)^{\frac{n}{2}}}{(h \beta \sigma)^{n}} \int_{-\infty}^{\infty} \prod_{a=1}^{n} d r_{a} \frac{1}{\sqrt{1+\sum_{a=1}^{n} r_{a}^{2}}} \\
& =\frac{(2 \pi)^{\frac{n}{2}}}{(h \beta \sigma)^{n}} \frac{2 \pi^{\frac{n}{2}}}{\Gamma\left(\frac{n}{2}\right)} \int_{0}^{\infty} d R \frac{R^{n-1}}{\sqrt{1+R^{2}}} \\
& =\frac{(2 \pi)^{\frac{n}{2}}}{(h \beta \sigma)^{n}} \frac{\pi^{\frac{n}{2}}}{\Gamma\left(\frac{n}{2}\right)} B\left(\frac{n}{2}, \frac{1-n}{2}\right) \\
& =\frac{2^{-\frac{n}{2}}}{(\hbar \beta \sigma)^{n} \sqrt{\pi}} \Gamma\left(\frac{1-n}{2}\right) .
\end{aligned}
$$

Since $F(n)=E\left[Z^{n}\right],(n \in \mathbf{C})$ in Eq. (14) is derived by FSNJ and $G(n)=E\left[Z^{n}\right],(n \in \mathbf{Z})$ in Eq. (15) by FJNS are also consistent, the replica analytic continuation is validated in this model where in Eq. (15) $r_{a}=q_{a} \sqrt{\beta m}$, $R^{2}=\sum_{a=1}^{n} r_{a}^{2}, B(p, q)=\frac{\Gamma(p) \Gamma(q)}{\Gamma(p+q)}$ and Eq. (12) are used.

\section{Classical Harmonic Oscillator Model with Chi Distribution}

We consider also the case where $\omega$ is distributed with a $\chi$ distribution, $P_{\chi}(\omega)$, with degrees of freedom $s$, defined as

$$
P_{\chi}(\omega)=\left\{\begin{array}{ll}
\frac{\omega^{s-1}}{2^{\frac{s-2}{2}} \Gamma\left(\frac{s}{2}\right)} e^{-\frac{\omega^{2}}{2}} & \omega>0 \\
0 & \text { otherwise }
\end{array} .\right.
$$

For FSNJ, when $n \in \mathbf{C}, F(n)=E\left[Z^{n}\right]$, is evaluated as

$$
\begin{aligned}
E\left[Z^{n}\right] & =\int_{0}^{\infty} d \omega P_{\chi}(\omega)\left(\frac{1}{\hbar \beta \omega}\right)^{n} \\
& =\frac{2^{-\frac{n}{2}}}{(\hbar \beta)^{n}} \frac{\Gamma\left(\frac{s-n}{2}\right)}{\Gamma\left(\frac{s}{2}\right)} .
\end{aligned}
$$

For FJNS, when $n \in \mathbf{Z}, G(n)=E\left[Z^{n}\right]$ is calculated as

$$
\begin{aligned}
E\left[Z^{n}\right] & =\frac{1}{h^{n}}\left(\frac{2 \pi m}{\beta}\right)^{\frac{n}{2}} \int_{-\infty}^{\infty} \prod_{a=1}^{n} d q_{a} \\
& \int_{0}^{\infty} d \omega \frac{\omega^{s-1}}{2^{\frac{s-2}{2}} \Gamma\left(\frac{s}{2}\right)} e^{-\frac{\omega^{2}}{2}\left(1+\beta m \sum_{a=1}^{n} q_{a}^{2}\right)} \\
= & \frac{(2 \pi)^{\frac{n}{2}}}{(h \beta)^{n}} \int_{-\infty}^{\infty} \prod_{a=1}^{n} d r_{a} \frac{1}{\left(1+\sum_{a=1}^{n} r_{a}^{2}\right)^{\frac{s}{2}}} \\
= & \frac{(2 \pi)^{\frac{n}{2}}}{(h \beta)^{n}} \frac{2 \pi^{\frac{n}{2}}}{\Gamma\left(\frac{n}{2}\right)} \int_{0}^{\infty} d R \frac{R^{n-1}}{\left(1+R^{2}\right)^{\frac{s}{2}}} \\
= & \frac{2^{-\frac{n}{2}}}{(\hbar \beta)^{n} \Gamma\left(\frac{n}{2}\right)} B\left(\frac{n}{2}, \frac{s-n}{2}\right) \\
= & \frac{2^{-\frac{n}{2}}}{(\hbar \beta)^{n}} \frac{\Gamma\left(\frac{s-n}{2}\right)}{\Gamma\left(\frac{s}{2}\right)} .
\end{aligned}
$$

$F(n)=E\left[Z^{n}\right],(n \in \mathbf{C})$ in Eq. (17) analyzed by FSNJ is consistent with $G(n)=E\left[Z^{n}\right],(n \in \mathbf{Z})$ in Eq. (18) analyzed by FJNS since these descriptions are consistent with $n \notin \mathbf{Z}$, and thus replica analytic continuation may hold in this model. Note that this model includes the models in Subsections III A and III B as special cases.

\section{Quantum Harmonic Oscillator Model with Exponential Distribution}

The models for which replica analytic continuation is satisfied are not limited to the classical harmonic oscillator model. Here, we discuss another commonly used analytical model, the quantum harmonic oscillator model. The Hamiltonian of the quantum harmonic oscillator model, $\mathcal{H}(k)$, is defined as

$$
\mathcal{H}(k)=\hbar \omega\left(k+\frac{1}{2}\right),
$$

where $k$ is the quantum number and $\omega$ is the positive angular frequency. In the case of a Boson, $k=0,1,2,3, \cdots$ and the partition function $Z$ in this model is calculated as

$$
\begin{aligned}
Z & =\sum_{k=0}^{\infty} e^{-\beta \mathcal{H}(k)} \\
& =\frac{e^{-\frac{\hbar \beta \omega}{2}}}{1-e^{-\hbar \beta \omega}} .
\end{aligned}
$$


Moreover, it is assumed that the angular frequency $\omega$ is distributed with the exponential distribution $P_{\mu}(\omega)$ :

$$
P_{\mu}(\omega)=\left\{\begin{array}{ll}
\frac{1}{\mu} e^{-\frac{\omega}{\mu}} & \omega>0 \\
0 & \text { otherwise }
\end{array} .\right.
$$

From this, for FSNJ, when $n \in \mathbf{C}, F(n)=E\left[Z^{n}\right]$ is evaluated as

$$
\begin{aligned}
E\left[Z^{n}\right] & =\int_{0}^{\infty} d \omega P_{\mu}(\omega) \frac{e^{-\frac{n \hbar \beta \omega}{2}}}{\left(1-e^{-\hbar \beta \omega}\right)^{n}} \\
& =\frac{1}{\hbar \beta \mu} \int_{0}^{1} d t t^{\frac{n}{2}+\frac{1}{\hbar \beta \mu}-1}(1-t)^{1-n-1} \\
& =\frac{1}{\hbar \beta \mu} B\left(\frac{n}{2}+\frac{1}{\hbar \beta \mu}, 1-n\right),
\end{aligned}
$$

where $t=e^{-\hbar \beta \omega}$ is used.

For FJNS, when $n \in \mathbf{Z}, G(n)=E\left[Z^{n}\right]$ is calculated as

$$
\begin{aligned}
E\left[Z^{n}\right] & =\sum_{\vec{k}=0}^{\infty} \int_{0}^{\infty} d \omega P_{\mu}(\omega) e^{-\hbar \beta \omega \sum_{a=1}^{n}\left(k_{a}+\frac{1}{2}\right)} \\
& =\sum_{\vec{k}=0}^{\infty} \frac{1}{\hbar \beta \mu\left(\frac{1}{\hbar \beta \mu}+\frac{n}{2}+\sum_{a=1}^{n} k_{a}\right)} \\
& =\sum_{m=0}^{\infty} \frac{1}{\hbar \beta \mu\left(\frac{1}{\hbar \beta \mu}+\frac{n}{2}+m\right)}\left(\begin{array}{c}
n-1+m \\
m
\end{array}\right) \\
& =\frac{1}{\hbar \beta \mu \Gamma(n)} \int_{0}^{\infty} d s \int_{0}^{\infty} d u u^{n-1} e^{-s\left(\frac{n}{2}+\frac{1}{\hbar \beta \mu}\right)-u+u e^{-s}} \\
& =\frac{1}{\hbar \beta \mu} \int_{0}^{1} d t t^{\frac{n}{2}+\frac{1}{\hbar \beta \mu}-1}(1-t)^{1-n-1} \\
& =\frac{1}{\hbar \beta \mu} B\left(\frac{n}{2}+\frac{1}{\hbar \beta \mu}, 1-n\right)
\end{aligned}
$$

where $\vec{k}=\left(k_{1}, k_{2}, \cdots, k_{n}\right)^{\mathrm{T}} \in \mathbf{Z}^{n}$ and the Kronecker delta $\delta(c, d)$ are used. Further

$$
\sum_{\vec{k}=0}^{\infty} \delta\left(m, \sum_{a=1}^{n} k_{a}\right)=\left(\begin{array}{c}
n-1+m \\
m
\end{array}\right),
$$

and $t=e^{-s}$ are employed. From this, $F(n)=$ $E\left[Z^{n}\right],(n \in \mathbf{C})$ in Eq. (22) analyzed by FSNJ is consistent with $G(n)=E\left[Z^{n}\right],(n \in \mathbf{Z})$ in Eq. (23) calculated by FJNS, indicating that replica analytic continuation also holds in this model.

\section{E. Random Field Ising Model}

In this subsection, Fermi's quantum harmonic oscillator, the Fermion, with $k=0,1$, is discussed using the Ising model as the equivalent representation. For convenience, we here accept the Ising model, the Hamiltonian of which is defined as

$$
\mathcal{H}(S)=-h S
$$

where $S(= \pm 1)$ indicates the Ising spin and $h$ is the external magnetic field. Then, the partition function $Z$ is estimated easily as

$$
\begin{aligned}
Z & =\sum_{S= \pm 1} e^{-\beta \mathcal{H}(S)} \\
& =2 \cosh \beta h .
\end{aligned}
$$

Furthermore, it is presumed that the external magnetic field $h$ is distributed with a Gaussian distribution with mean $\mu$ and variance $\sigma^{2}, P_{\mu, \sigma^{2}}(h)$ :

$$
P_{\mu, \sigma^{2}}(h)=\frac{e^{-\frac{(h-\mu)^{2}}{2 \sigma^{2}}}}{\sqrt{2 \pi \sigma^{2}}} .
$$

Then, for FSNJ, in the case of $n \in \mathbf{C}, F(n)=E\left[Z^{n}\right]$ is solved as

$$
\begin{aligned}
E\left[Z^{n}\right] & =\int_{-\infty}^{\infty} d h P_{\mu, \sigma^{2}}(h)(2 \cosh \beta h)^{n} \\
& =\int_{-\infty}^{\infty} D x(2 \cosh (\beta \mu+\beta \sigma x))^{n},
\end{aligned}
$$

where $D x=\frac{d x}{\sqrt{2 \pi}} e^{-\frac{x^{2}}{2}}$ is employed.

For FJNS, when $n \in \mathbf{Z}, G(n)=E\left[Z^{n}\right]$ is derived as

$$
\begin{aligned}
E\left[Z^{n}\right] & =\sum_{\vec{S} \in\{ \pm 1\}^{n}} \int_{-\infty}^{\infty} d h P_{\mu, \sigma^{2}}(h) e^{\beta h \sum_{a=1}^{n} S_{a}} \\
& =\sum_{\vec{S} \in\{ \pm 1\}^{n}} \exp \left(\beta \mu \sum_{a=1}^{n} S_{a}+\frac{\beta^{2} \sigma^{2}}{2}\left(\sum_{a=1}^{n} S_{a}\right)^{2}\right) \\
& =\sum_{m=0}^{n} e^{\beta \mu(2 m-n)+\frac{\beta^{2} \sigma^{2}(2 m-n)^{2}}{2}}\left(\begin{array}{c}
n \\
m
\end{array}\right) \\
& =\int_{-\infty}^{\infty} D x e^{-n \beta \mu-n \beta \sigma x}\left(1+e^{2 \beta \mu+2 \beta \sigma x}\right)^{n} \\
& =\int_{-\infty}^{\infty} D x(2 \cosh (\beta \mu+\beta \sigma x))^{n},
\end{aligned}
$$

where $\vec{S}=\left(S_{1}, S_{2}, \cdots, S_{n}\right)^{\mathrm{T}} \in\{ \pm 1\}^{n}$ is used and the notation $\sum_{\vec{S} \in\{ \pm 1\}^{n}}$ means a sum over the whole configuration of $\vec{S}$. Furthermore, $\int_{-\infty}^{\infty} D x e^{\theta x}=e^{\frac{\theta^{2}}{2}}$ and

$$
\sum_{\vec{S} \in\{ \pm 1\}^{n}} \delta\left(2 m-n, \sum_{a=1}^{n} S_{a}\right)=\left(\begin{array}{c}
n \\
m
\end{array}\right),
$$

are used. From Eq. (28) and Eq. (29), $F(n)=$ $E\left[Z^{n}\right],(n \in \mathbf{C})$ for FSNJ and $G(n)=E\left[Z^{n}\right],(n \in \mathbf{Z})$ for FJNS are equivalent, showing that replica analytic continuation holds for this model.

\section{F. Potential Energy for Near Earth Gravity}

In this subsection, we discuss whether replica analytic continuation holds using a classical physics model that is 
not often used in statistical physics, the potential energy for near Earth gravity. For $g$, the gravitational acceleration, the Hamiltonian $\mathcal{H}(x)$ is defined by the potential energy of the particle (its mass $m$ ) at position $x, m g x$, where the potential energy at origin is regarded as 0 :

$$
\mathcal{H}(x)=\operatorname{mgx} .
$$

We here consider the canonical ensemble of this model whose range is the set of non-negative real numbers. The partition function of this model $Z$ is calculated as

$$
\begin{aligned}
Z & =\frac{1}{h} \int_{0}^{\infty} d x e^{-\beta \mathcal{H}(x)} \\
& =\frac{1}{h \beta m g} .
\end{aligned}
$$

Here it is assumed that the mass $m$ is randomly distributed with the Gamma distribution with shape parameter $\tau \in \mathbf{R}, P_{\tau}(m)$ :

$$
P_{\tau}(m)=\left\{\begin{array}{ll}
\frac{m^{\tau-1} e^{-m}}{\Gamma(\tau)} & m>0 \\
0 & \text { otherwise }
\end{array} .\right.
$$

Using this distribution, for FSNJ, in the case of $n \in \mathbf{C}$, $F(n)=E\left[Z^{n}\right]$ is solved as

$$
\begin{aligned}
E\left[Z^{n}\right] & =\int_{0}^{\infty} d m P_{\tau}(m)\left(\frac{1}{h \beta g m}\right)^{n} \\
& =\frac{1}{(h \beta g)^{n}} \frac{\Gamma(\tau-n)}{\Gamma(\tau)} .
\end{aligned}
$$

For FJNS, when $n \in \mathbf{Z}, G(n)=E\left[Z^{n}\right]$ is described as

$$
\begin{aligned}
E\left[Z^{n}\right] & =\frac{1}{h^{n}} \int_{0}^{\infty} \prod_{a=1}^{n} d x_{a} \int_{0}^{\infty} d m P_{\tau}(m) e^{-\beta m g \sum_{a=1}^{n} x_{a}} \\
& =\frac{1}{(h \beta g)^{n}} \int_{0}^{\infty} \prod_{a=1}^{n} d r_{a} \frac{1}{\left(1+\sum_{a=1}^{n} r_{a}\right)^{\tau}}
\end{aligned}
$$

where $r_{a}=\beta g x_{a}$ is used. Moreover, when $k>1$,

$$
\int_{0}^{\infty} \frac{d r}{(\alpha+r)^{k}}=\frac{1}{(k-1) \alpha^{k-1}},
$$

is obtained. Using this formula, we can recursively solve $G(n)$ as

$$
\begin{aligned}
E\left[Z^{n}\right]= & \frac{1}{(h \beta g)^{n}} \frac{1}{(\tau-1)} \frac{1}{(\tau-2)} \cdots \frac{1}{(\tau-l)} \\
& \int_{0}^{\infty} \prod_{a=1}^{n-l} d r_{a} \frac{1}{\left(1+\sum_{a=1}^{n-l} r_{a}\right)^{\tau-l}} \\
= & \frac{1}{(h \beta g)^{n}} \frac{1}{(\tau-1)} \frac{1}{(\tau-2)} \cdots \frac{1}{(\tau-n)} \\
= & \frac{1}{(h \beta g)^{n}} \frac{\Gamma(\tau-n)}{\Gamma(\tau)} .
\end{aligned}
$$

$F(n)=E\left[Z^{n}\right],(n \in \mathbf{C})$ in Eq. (34) analyzed by FSNJ and $G(n)=E\left[Z^{n}\right],(n \in \mathbf{Z})$ in Eq. (37) analyzed by FJNS are consistent, and thus replica analytic continuation is guaranteed in this model.

\section{G. Moment of Chi-Squared Distribution}

In this subsection we discuss whether replica analytic continuation holds for a model which is not used in physics, namely, where the random variable $Z$ is distributed with a $\chi^{2}$ distribution with degrees of freedom $m, P_{\chi^{2}(m)}(Z)$ :

$$
P_{\chi^{2}(m)}(Z)= \begin{cases}\frac{1}{2^{\frac{m}{2}} \Gamma\left(\frac{m}{2}\right)} Z^{\frac{m}{2}-1} e^{-\frac{Z}{2}} & Z>0 \\ 0 & \text { otherwise }\end{cases}
$$

where $Z$ of this $\chi^{2}$ distribution is the sum of the squares of $m$ random variables, $x_{1}, \cdots, x_{m}$, which are independently and identically drawn from the standard normalized distribution, that is, $Z=\sum_{i=1}^{m} x_{i}^{2}$. We regard the sum of squares $Z=\sum_{i=1}^{m} x_{i}^{2}$ to be the partition function.

For FSNJ, since the distribution of $Z$ is already known, that is, we can consider the thermodynamical average to be determined, when $n \in \mathbf{C}, F(n)=E\left[Z^{n}\right]$, is evaluated as

$$
\begin{aligned}
E\left[Z^{n}\right] & =\int_{0}^{\infty} d Z P_{\chi^{2}(m)}(Z) Z^{n} \\
& =\frac{2^{n} \Gamma\left(\frac{m}{2}+n\right)}{\Gamma\left(\frac{m}{2}\right)} .
\end{aligned}
$$

For FJNS, since $Z$ is constructed from the sum of $m$ independently and identically distributed variables, that is, $Z=\sum_{i=1}^{m} x_{i}^{2}$, when $n \in \mathbf{Z}, G(n)=E\left[Z^{n}\right]$ is expanded as

$$
\begin{aligned}
E\left[Z^{n}\right] & =\int_{-\infty}^{\infty} \prod_{i=1}^{m} D x_{i}\left(\sum_{i=1}^{m} x_{i}^{2}\right)^{n} \\
& =\int_{-\infty}^{\infty} \prod_{i=1}^{m} D x_{i} \sum_{\vec{k}=0}^{n} \frac{n !}{\prod_{i=1}^{m} k_{i} !} \prod_{i=1}^{m} x_{i}^{2 k_{i}} \delta\left(n, \sum_{i=1}^{m} k_{i}\right) \\
& =2^{n} n ! \sum_{\vec{k}=0}^{n} \delta\left(n, \sum_{i=1}^{m} k_{i}\right) \prod_{i=1}^{m} \frac{\Gamma\left(k_{i}+\frac{1}{2}\right)}{\Gamma\left(\frac{1}{2}\right) \Gamma\left(k_{i}+1\right)},
\end{aligned}
$$

where $\vec{k}=\left(k_{1}, \cdots, k_{m}\right)^{\mathrm{T}} \in\{0,1,2, \cdots, n\}^{m}$ and

$$
\int_{-\infty}^{\infty} D x x^{2 k}=\frac{2^{k}}{\Gamma\left(\frac{1}{2}\right)} \Gamma\left(k+\frac{1}{2}\right),
$$

are used. We can verify that Eq. (39) and Eq. (40) are consistent by using mathematical induction. For convenience, the right hand sides of Eq. (39) and Eq. (40) are rewritten as

$$
\begin{aligned}
& F(m, n)=\frac{2^{n} \Gamma\left(\frac{m}{2}+n\right)}{\Gamma\left(\frac{m}{2}\right)} \\
& G(m, n)=2^{n} n ! \sum_{\vec{k}=0}^{n} \delta\left(n, \sum_{i=1}^{m} k_{i}\right) \prod_{i=1}^{m} \frac{\Gamma\left(k_{i}+\frac{1}{2}\right)}{\Gamma\left(\frac{1}{2}\right) \Gamma\left(k_{i}+1\right)} .
\end{aligned}
$$


When $m=1$, for any $n \in \mathbf{Z}$,

$$
\begin{aligned}
F(1, n) & =\frac{2^{n} \Gamma\left(n+\frac{1}{2}\right)}{\Gamma\left(\frac{1}{2}\right)} \\
G(1, n) & =2^{n} n ! \sum_{k_{1}=0}^{n} \delta\left(n, k_{1}\right) \frac{\Gamma\left(k_{1}+\frac{1}{2}\right)}{\Gamma\left(\frac{1}{2}\right) \Gamma\left(k_{1}+1\right)} \\
& =2^{n} n ! \frac{\Gamma\left(n+\frac{1}{2}\right)}{\Gamma\left(\frac{1}{2}\right) \Gamma(n+1)} \\
& =2^{n} \frac{\Gamma\left(n+\frac{1}{2}\right)}{\Gamma\left(\frac{1}{2}\right)} .
\end{aligned}
$$

This shows that $F(1, n)=G(1, n)$ holds.

Next, we set

$$
\begin{aligned}
& F(s, t)=\frac{2^{t} \Gamma\left(\frac{s}{2}+t\right)}{\Gamma\left(\frac{s}{2}\right)} \\
& G(s, t)=2^{t} t ! \sum_{\vec{k}=0}^{t} \delta\left(t, \sum_{i=1}^{s} k_{i}\right) \prod_{i=1}^{s} \frac{\Gamma\left(k_{i}+\frac{1}{2}\right)}{\Gamma\left(\frac{1}{2}\right) \Gamma\left(k_{i}+1\right)}
\end{aligned}
$$

and assume that $F(s, t)=G(s, t)$ holds when $m=s \in \mathbf{Z}$ for any $t \in \mathbf{Z}$. Then, when $m=s+1$,

$$
\begin{aligned}
& G(s+1, t) \\
= & 2^{t} t ! \sum_{k_{s+1}=0}^{t} \sum_{k_{s}=0}^{t} \sum_{k_{s-1}=0}^{t} \cdots \sum_{k_{1}=0}^{t} \\
& \delta\left(t, \sum_{i=1}^{s} k_{i}+k_{s+1}\right) \frac{\Gamma\left(k_{s+1}+\frac{1}{2}\right)}{\Gamma\left(\frac{1}{2}\right) \Gamma\left(k_{s+1}+1\right)} \\
& \prod_{i=1}^{s} \frac{\Gamma\left(k_{i}+\frac{1}{2}\right)}{\Gamma\left(\frac{1}{2}\right) \Gamma\left(k_{i}+1\right)} \\
= & 2^{t} t ! \sum_{k_{s+1}=0}^{t} \frac{\Gamma\left(k_{s+1}+\frac{1}{2}\right)}{\Gamma\left(\frac{1}{2}\right) \Gamma\left(k_{s+1}+1\right)} \frac{2^{k_{s+1}-t}}{\Gamma\left(t-k_{s+1}+1\right)} \\
& \left\{2^{t-k_{s+1}\left(t-k_{s+1}\right) ! \sum_{k_{s}=0}^{t-k_{s+1}} \sum_{k_{s-1}=0}^{t-k_{s+1}} \sum_{k_{1}=0}^{t-k_{s+1}}}\right. \\
& \left.\delta\left(t-k_{s+1}, \sum_{i=1}^{s} k_{i}\right) \prod_{i=1}^{s} \frac{\Gamma\left(k_{i}+\frac{1}{2}\right)}{\Gamma\left(\frac{1}{2}\right) \Gamma\left(k_{i}+1\right)}\right\} \\
= & 2^{t} t ! \sum_{k_{s+1}=0}^{t} \frac{\Gamma\left(k_{s+1}+\frac{1}{2}\right)}{\Gamma\left(\frac{1}{2}\right) \Gamma\left(k_{s+1}+1\right)} \frac{2^{k_{s+1}-t}}{\Gamma\left(t-k_{s+1}+1\right)} \\
& G\left(s, t-k_{s+1}\right) .
\end{aligned}
$$

Further, from the assumption of mathematical induction, since $G\left(s, t-k_{s+1}\right)=F\left(s, t-k_{s+1}\right)$ holds, we have

$$
\begin{aligned}
& G(s+1, t) \\
= & 2^{t} t ! \sum_{k_{s+1}=0}^{t} \frac{\Gamma\left(k_{s+1}+\frac{1}{2}\right)}{\Gamma\left(\frac{1}{2}\right) \Gamma\left(k_{s+1}+1\right)} \frac{2^{k_{s+1}-t}}{\Gamma\left(t-k_{s+1}+1\right)} \\
& 2^{t-k_{s+1}} \frac{\Gamma\left(\frac{s}{2}+t-k_{s+1}\right)}{\Gamma\left(\frac{s}{2}\right)} \\
= & \frac{2^{t}}{\Gamma\left(\frac{1}{2}\right) \Gamma\left(\frac{s}{2}\right)} \sum_{k_{s+1}=0}^{t} \frac{t !}{k_{s+1} !\left(t-k_{s+1}\right) !} \\
& \Gamma\left(k_{s+1}+\frac{1}{2}\right) \Gamma\left(t-k_{s+1}+\frac{s}{2}\right) \\
= & \frac{2^{t}}{\Gamma\left(\frac{1}{2}\right) \Gamma\left(\frac{s}{2}\right)} \sum_{k=0}^{t} \frac{t !}{k !(t-k) !} \\
& \int_{0}^{\infty} d u u^{k+\frac{1}{2}-1} e^{-u} \int_{0}^{\infty} d v v^{t-k+\frac{s}{2}-1} e^{-v} \\
= & \frac{2^{t}}{\Gamma\left(\frac{1}{2}\right) \Gamma\left(\frac{s}{2}\right)} \int_{0}^{\infty} d u u^{\frac{1}{2}-1} e^{-u} \int_{0}^{\infty} d v v^{\frac{s}{2}-1} e^{-v} \\
& \sum_{k=0}^{t} \frac{t !}{k !(t-k) !} u^{k} v^{t-k} \\
= & 2^{t} \int_{0}^{\infty} d u \frac{u^{\frac{1}{2}-1} e^{-u}}{\Gamma\left(\frac{1}{2}\right)} \int_{0}^{\infty} d v \frac{v^{\frac{s}{2}-1} e^{-v}}{\Gamma\left(\frac{s}{2}\right)} \\
& (u+v)^{t} .
\end{aligned}
$$

Setting $u=\frac{x}{2}$ and $v=\frac{y}{2}$ we obtain

$$
\begin{aligned}
& G(s+1, t) \\
= & 2^{t} \int_{0}^{\infty} d x \frac{x^{\frac{1}{2}-1} e^{-\frac{x}{2}}}{\Gamma\left(\frac{1}{2}\right) 2^{\frac{1}{2}}} \int_{0}^{\infty} d y \frac{y^{\frac{s}{2}-1} e^{-\frac{y}{2}}}{\Gamma\left(\frac{s}{2}\right) 2^{\frac{s}{2}}}\left(\frac{x+y}{2}\right)^{t} \\
= & \int_{0}^{\infty} d x P_{\chi^{2}(1)}(x) \int_{0}^{\infty} d y P_{\chi^{2}(s)}(y)(x+y)^{t} \\
= & \int_{0}^{\infty} d z P_{\chi^{2}(s+1)}(z) z^{t} \\
= & \frac{2^{t} \Gamma\left(\frac{s+1}{2}+t\right)}{\Gamma\left(\frac{s+1}{2}\right)} \\
= & F(s+1, t) .
\end{aligned}
$$

Thus, $F(s+1, t)=G(s+1, t)$ holds. Note that we use the property that the sum of the random variable $x$, which is drawn from the $\chi^{2}$ distribution with 1 degree of freedom, and the random variable $y$, which is drawn from the $\chi^{2}$ distribution with degrees of freedom $s$, so that $z=x+y$ is distributed with a $\chi^{2}$ distribution with degrees of freedom $s+1$. From this induction, it is verified that $F(m, n)=G(m, n)$ holds. Finally, since both descriptions, $F(n)=E\left[Z^{n}\right],(n \in \mathbf{C})$ for FSNJ and $G(n)=E\left[Z^{n}\right],(n \in \mathbf{Z})$ for FJNS, are also consistent with each other, we have shown that replica analytic continuation holds in this model. 


\section{H. Other Toy Models}

Lastly, we introduce other toy models which satisfy replica analytic continuation:

1. From the partition function $Z=\int_{-\infty}^{\infty} D x e^{x \omega}=$ $e^{\frac{\omega^{2}}{2}}$ and $P_{\sigma}(\omega)$ in Eq. (13), $F(n)=E\left[Z^{n}\right]=$ $\left(1-n \sigma^{2}\right)^{-\frac{1}{2}}$ is obtained. Since $x_{a} \sim N(0,1),(a=$ $1, \cdots, n)$, it is well known that $s=\sigma \sum_{a=1}^{n} x_{a}$ is distributed with a Gaussian distribution with mean 0 and variance $n \sigma^{2}$. Using $s$, we can also easily analyze $G(n)$ for FJNS.

2. We consider a random variable $Z$ from the $F$ distribution with degrees of freedom $s$ and $t$ :

$$
P(Z)=\left\{\begin{array}{ll}
\frac{s^{\frac{s}{2}} t^{\frac{t}{2}}}{B\left(\frac{s}{2}, \frac{t}{2}\right)} \frac{Z^{\frac{s-2}{2}}}{(s Z+t)^{\frac{s+t}{2}}} & Z>0 \\
0 & Z \leq 0
\end{array} .\right.
$$

Then, $F(n)=E\left[Z^{n}\right],(n \in \mathbf{C})$ is calculated as follows.

$$
E\left[Z^{n}\right]=\left(\frac{t}{s}\right)^{n} \frac{\Gamma\left(\frac{s}{2}+n\right) \Gamma\left(\frac{t}{2}-n\right)}{\Gamma\left(\frac{s}{2}\right) \Gamma\left(\frac{t}{2}\right)}
$$

We can regard the random variable $Z$ as being the ratio between the squared average of $s$ random variables $x_{k},(k=1, \cdots, s)$ which are independently and identically distributed with a standard normal distribution, and the squared average of $t$ random variables $y_{l},(l=1, \cdots, t)$ which are also independently and identically distributed with a standard normal distribution as follows:

$$
Z=\frac{\frac{1}{s} \sum_{k=1}^{s} x_{k}^{2}}{\frac{1}{t} \sum_{l=1}^{t} y_{l}^{2}} .
$$

Thus, $G(n)$ is also easily solved using the mathematical induction in Subsection III G.

\section{Discussion and Open Problems}

As stated above, in this study we have examined replica analytic continuation, the mathematical basis behind the replica trick, one of the two steps that comprise replica analysis. Some of the concerns about the reliability of replica analysis are based on doubts about the accuracy of the replica trick. When applying replica analysis, both the replica trick and the thermodynamical limit are used, and hence it can be hard to unravel any inaccuracies in replica analysis. Therefore, we have applied replica analysis to some physical or statistical models for which the thermodynamical limit need not be applied, so that we can test the accuracy of replica analytical continuation alone, thereby definitively addressing part of the basis of replica analysis.
In this paper, although we considered only a few examples due to the limitations of space, it is clear from the results of the models for which we can apply both analytical procedures, FSNJ and FJNS, that we can assess $F(n)=E\left[Z^{n}\right],(n \in \mathbf{C})$ and $G(n)=E\left[Z^{n}\right],(n \in \mathbf{Z})$ without approximation and can verify that replica analytic continuation is guaranteed in these models. We note that a more complete analysis would examine a counterexample to the case of replica analytical continuation in mathematical models which can be analyzed without approximation. Thus, while the analysis in this paper does not provide a universal mathematical proof of replica analysis, the positive examples detailed in this paper lead us to the following conjecture:

\section{Conjecture on Replica Analytic Continuation:}

With respect to models where we can assess the FSNJ and FJNS approaches without approximation, for $F(n)=E\left[Z^{n}\right],(n \in \mathbf{C})$ analyzed by the FSNJ procedure and $G(n)=E\left[Z^{n}\right],(n \in \mathbf{Z})$ analyzed by the FJNS procedure, when $n \in \mathbf{Z}$, $F(n)=G(n)$ is always satisfied. In addition, $G(n)$ is regarded as an analytical function of the replica number $n$, and we assume that $G(n)$ is also described as an analytical function of $n$ when $n \notin \mathbf{Z}$, so then if $F(n)=G(n),(n \notin \mathbf{Z})$ holds, the replica analytic continuation for this model is guaranteed.

Namely, given the positive results of our study, it can now be expected that any doubts about the accuracy of replica analysis will not be directed at replica analytic continuation. Hence any questions regarding replica analysis reported in previous works are likely to be based on more complicated aspects of replica analysis, particularly when the thermodynamical limit and the expansion on order parameters are included. This paper has taken the approach of decomposing replica analysis into its composite components, allowing the mathematical properties of each component to be investigated in order to clarify the mathematical structure of replica analysis. However, it is also important to examine problems where the components are used in combination.

\section{CONCLUSION AND FUTURE WORKS}

In this work, to explore the roots of questions on the validity of replica analysis, we applied replica analytic continuation to analyze harmonic oscillator models (classical and quantum) and the Ising model, which do not need consideration of the thermodynamical limit, and validated the effectiveness of replica analytic continuation. Although the present paper handles only a few examples, we discussed whether replica analytic continuation holds with respect to toy models for which it is possible to solve $F(n)=E\left[Z^{n}\right],(n \in \mathbf{C})$ and $G(n)=E\left[Z^{n}\right],(n \in \mathbf{Z})$ using the FSNJ and FJNS approaches without approximations. Although we cannot 
provide a universal proof on the validity of replica analysis in this paper, our goal was to formulate a conjecture for replica analytic continuation. We do not discuss the thermodynamical limit and order parameter expansion, though these have already been guaranteed theoretically and practically, and are not particularly under question. However, considering more complicated cases will be difficult [33] as even if each analytical technique can be proved individually, they are complicated when combined.

In future work we need to discuss whether replica analytic continuation holds for physical or statistical models of many-body systems using FSNJ and FJNS without approximations. Although we considered a few positive examples in this paper, it is preferable to prove the conjecture of replica analytic continuation in a general form. Furthermore, although we discussed replica analytic continuation using the versatile properties of the Gamma function, other special functions could further support the proof of replica analytic continuation.

\section{ACKNOWLEDGEMENTS}

The author appreciates the helpful comments offered by Y. Kabashima, T. Tanaka and K. Kobayashi. The work is partly supported by Grant-in-Aid Nos. 24710169 and 15K20999; the President Project for Young Scientists at Akita Prefectural University; grant No. 50 of the National Institute of Informatics, Japan; grant No. 5 of the Japan Institute of Life Insurance; a grant from the Institute of Economic Research Foundation; Kyoto University; grant No. 1414 of the Zengin Foundation for Studies in Economics and Finance; grant No. 2068 of the Institute of Statistical Mathematics; research project of Mitsubishi UFJ Trust Scholarship Foundation; and grant No. 2 of the Kampo Foundation.

\section{Appendix A: Calculation of $E[\log Z]$}

Here we discuss the expectation of the logarithm of the partition function $E[\log Z]$. It is well-known that the Gamma function of $z, \Gamma(z)=\int_{0}^{\infty} d t t^{z-1} e^{-t}$ is described as

$$
\Gamma(z)=\frac{1}{z} e^{-\gamma z} \prod_{k=1}^{\infty}\left(\frac{k}{k+z}\right) e^{\frac{z}{k}}
$$

Using this description, the derivative of the logarithm function of the Gamma function with respect to $z$,

$$
\begin{aligned}
\psi(z) & =\frac{\partial}{\partial z} \log \Gamma(z) \\
& =-\gamma-\frac{1}{z}+\sum_{k=1}^{\infty}\left(\frac{1}{k}-\frac{1}{k+z}\right),
\end{aligned}
$$

is easily calculated where the Euler constant $\gamma$ is defined as

$$
\begin{aligned}
\gamma & =\lim _{L \rightarrow \infty}\left(\sum_{k=1}^{L} \frac{1}{k}-\log L\right) \\
& \simeq 0.57721 .
\end{aligned}
$$

We call $\psi(z)$ the digamma function. Especially for $z \in \mathbf{Z}$, the digamma function is formulated as follows;

$$
\begin{gathered}
\psi(z)=-\gamma+\sum_{k=1}^{z-1} \frac{1}{k}, \\
\psi\left(z+\frac{1}{2}\right)=-\gamma-2 \log 2+\sum_{k=1}^{z} \frac{1}{k-\frac{1}{2}} .
\end{gathered}
$$

From which we have,

$$
\begin{aligned}
\psi(1) & =-\gamma \\
\psi\left(\frac{1}{2}\right) & =-\gamma-2 \log 2
\end{aligned}
$$

From these settings, the expectation of the logarithm of the partition function $E[\log Z]$ in each case is solved as follows:

\section{Expectation of $\log Z$ in Section III A}

$$
E[\log Z]=\frac{\gamma}{2}-\frac{1}{2} \log 2-\log (\hbar \beta)
$$

2. Expectation of $\log Z$ in Section III B

$$
E[\log Z]=\frac{\gamma}{2}+\frac{1}{2} \log 2-\log (\hbar \beta \sigma)
$$

3. Expectation of $\log Z$ in Section III C

$$
E[\log Z]=-\frac{1}{2} \psi\left(\frac{s}{2}\right)-\frac{1}{2} \log 2-\log (\hbar \beta),
$$

\section{Expectation of $\log Z$ in Section III D}

$$
\begin{aligned}
E[\log Z] & =\frac{1}{2} \psi\left(\frac{1}{\hbar \beta \mu}\right)+\frac{1}{2} \psi\left(1+\frac{1}{\hbar \beta \mu}\right)-\psi(1) \\
& =-\frac{\hbar \beta \mu}{2}+\sum_{k=1}^{\infty}\left(\frac{1}{k}-\frac{1}{k+\frac{1}{\hbar \beta \mu}}\right),
\end{aligned}
$$




\section{Expectation of $\log Z$ in Section III E}

$$
E[\log Z]=\int_{-\infty}^{\infty} D x \log 2 \cosh (\beta \mu+\beta \sigma x)
$$

\section{Expectation of $\log Z$ in Section III $\mathbf{F}$}

$$
E[\log Z]=-\log (h \beta g)-\psi(\tau)
$$

\section{Expectation of $\log Z$ in Section III G}

$$
E[\log Z]=\log 2+\psi\left(\frac{m}{2}\right)
$$

\section{Expectation of $\log Z$ of 1st Example in Section III H}

$$
E[\log Z]=\frac{\sigma^{2}}{2}
$$

\section{Expectation of $\log Z$ of 2nd Example in Section} III H

$$
E[\log Z]=\log \frac{t}{s}+\psi\left(\frac{s}{2}\right)-\psi\left(\frac{t}{2}\right),
$$

[1] M. Mézard, G. Parisi, M. A. Virasoro, Spin glass theory and beyond (World Scientific Publishing, 1987).

[2] H. Nishimori, Statistical physics of spin glasses and information processing (Oxford University Press, 2001).

[3] M. Mézard, A. Montanari, Information, Physics, and Computation (Oxford University Press, 2009).

[4] S. F. Edwards, P. W. Anderson, J. Phys. F, 5, 965 (1975).

[5] D. Sherrington, S. Kirkpatrik, Phys. Rev. Let., 35, 1792 (1975).

[6] S. Kirkpatrik, D. Sherrington, Phys. Rev. B, 17, 4384 (1978).

[7] D. J. Amit, H. Gutfreund, H. Sompolinsky, Phys. Rev. A, 32, 1007 (1985).

[8] D. J. Amit, H. Gutfreund, H. Sompolinsky, Phys. Rev. Let., 55, 1530 (1985).

[9] E. Gardner, J. Phys. A, 21, 257 (1988).

[10] Y. Kabashima, D. Saad, J. Phys. A. 37, R1 (2004).

[11] T. Tanaka, IEEE on IT, 48, 2888 (2002).

[12] S. Ciliberti M. Mézard, Euro. Phys. J. B, 27, 175 (2007).

[13] T. Shinzato, PLoS One, 10, e0133846 (2015).

[14] M. Kac, Unpublished. Trondheim Theoretical Physics Seminar, Nordita Publ., No. 286 (1968).

[15] T.-F. F. Lin, J. Math. Phys. 11, 1584 (1970).

[16] S. F. Edwards, Proc. 4th Int. Conf. Amor. Mat., 279, Wiley, New York (1970).
[17] S. F. Edwards, Polymer Networks: Structural and Mechanical Properties, Plenum Press, New York (1971).

[18] V. J. Emery, Phys. Rev. B, 11, 239 (1975).

[19] D. Jasnow, M. E. Fisher, Phys. Rev. B, 13, 1112 (1976).

[20] G. Grinstein, A. Luther, Phys. Rev. B, 13, 1329 (1976).

[21] T. M. Cover, IEEE Trans. Electron. Comput., 14326 (1965).

[22] S. S. Venkatesh, AIP, Conf. Proc., 151, 440 (1986).

[23] T. Shinzato, Y. Kabashima, J. Phys. A, 41, 324013 (2008).

[24] J. R. L. Almeida, D. J. Thouless, J. Phys. A, 11, 983 (1978).

[25] G. Parisi, J. Phys. A, 13, 1101 (1980).

[26] G. Parisi, J. Phys. A, 13, L115 (1980).

[27] K. Ogure, Y. Kabashima, Prog. Theor. Phys., 111, 661 (2004).

[28] K. Ogure, Y. Kabashima, Prog. Theor. Phys. Suppl., 157, 103 (2005).

[29] T. Tanaka, Interdis. Info. Sci., 13, 17 (2007).

[30] E. Artin, The Gamma Function (Dover Publications, 2015).

[31] G. E. Andrews, R. Askey, R. Roy, Special Functions (Cambridge University Press, 2001).

[32] N. N. Levedev, Special Functions 65 Their Applications (Dover Publications, 1972).

[33] P. W. Anderson, Science, 177, 393 (1972). 